\title{
Cystic fibrosis: the conductance regulator, ceramides, and possible treatments
}

\author{
Friedrich C. Luft $^{1}$
}

Published online: 22 August 2017

(C) Springer-Verlag GmbH Germany 2017

Cystic fibrosis (CF) is the most common autosomal-recessive genetic disorder in man. The disease affects not only the lungs, but also the pancreas, liver, kidneys, and intestine. Long-term clinical issues include difficulty breathing and coughing up thick mucus, which promotes frequent lung infections. Other signs and symptoms may include sinus infections, poor growth, fatty stools, clubbing of the fingers and toes, and infertility in male patients. Earlier, patients died in childhood, although modern treatments have completely altered that state-of-affairs. Depending upon the mutations, the patients may have varying degrees of symptoms [1].

A mutation in the gene, cystic fibrosis transmembrane conductance regulator (CFTR) causes CF. Over 1500 CFTR mutations have been described, although the most common mutation, $\Delta \mathrm{F} 508$, is a deletion of three nucleotides that results in a loss of the amino acid phenylalanine at the 508th position on the protein. This mutation accounts for two thirds (66-70\%) of CF cases worldwide [2]. The CFTR protein is anchored to the outer membrane of cells in the sweat glands, lungs, pancreas, and all other exocrine glands in the body. CFTR acts as a channel and is responsible for controlling the movement of chloride from inside to outside of cells. The damage in CF is caused by blockage of narrow passageways in affected organs with thickened secretions.

The lungs of CF patients are colonized and infected by bacteria from an early age. These bacteria thrive in the altered mucus, which collects in the small airways of the lungs. This mucus leads to the formation of bacterial microenvironments,

Friedrich C. Luft

luft@charite.de

1 Experimental and Clinical Research Center, Max-Delbrück Center for Molecular Medicine and Charité Medical Faculty, Lindenberger Weg 80, 13125 Berlin, Germany termed biofilms, which are difficult for immune cells and antibiotics to penetrate. Viscous secretions and persistent respiratory infections, commonly Pseudomonas aeruginosa, repeatedly damage the lung by gradually remodeling the airways, which makes infection even more difficult to eradicate (Fig. 1) [3].

Sphingomyelin is a type of sphingolipid found in animal cell membranes, especially in the membranous myelin sheath that surrounds nerve cell axons. Sphingomyelin consists of a phosphocholine head group, a sphingosine, and a fatty acid. Sphingomyelin is enriched at the plasma membrane with a greater concentration on the outer than the inner leaflet. The sphingomyelinase pathway uses an enzyme to break down sphingomyelin in the cell membrane and release ceramide. Ceramides are a family of waxy lipid molecules, composed of sphingosine and a fatty acid. Contrary to previous assumptions that ceramides and other sphingolipids found in cell membrane were purely supporting structural elements, ceramide can participate in a variety of cellular signaling events. Examples include regulating differentiation, proliferation, and programmed cell death [4]. Lipid rafts are plasma membrane microdomains that are highly enriched in cholesterol and sphingolipids. Stimulation of CD95 or CD40 receptors activates sphingomyelinase in lipid rafts. As a result, ceramides are produced through the degradation of sphingomyelins. Sphingolipids have been strongly implicated in lung growth, maintenance, and repair [5].

Ceramide accumulation has been implicated in the pathogenesis of CF. Ceramide accumulates in the lungs of CF patients, causing inflammation and an increased susceptibility to bacterial infections. The possibility has been raised that interfering with sphingolipids could be of therapeutic relevance in $\mathrm{CF}$ patients [6]. Indeed, fenretinide (4-hydroxy phenylretinamide) is a synthetic retinoid derivative, related to vitamin A. Fenretinide has been investigated for potential use in 
A CF lung pathology

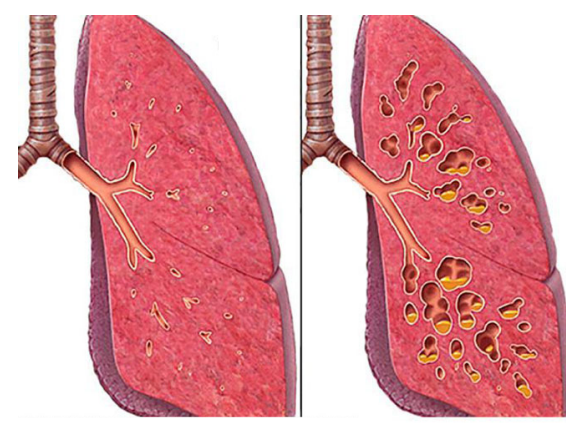

\section{Pulmonary epithelium}

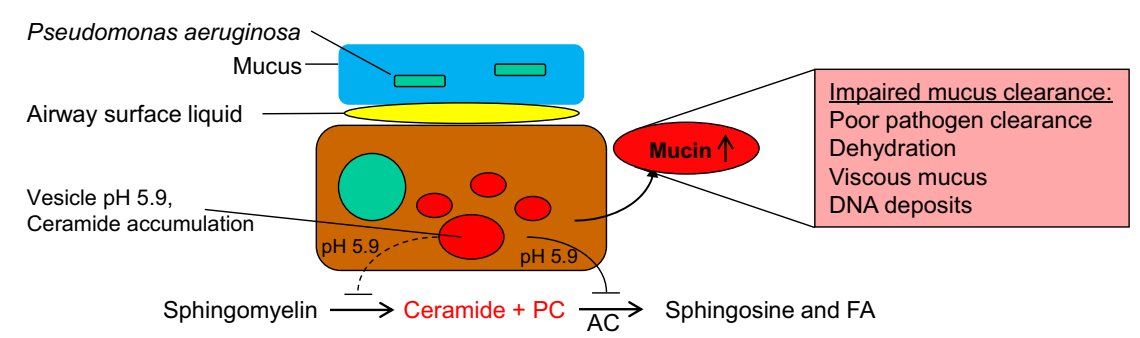

Fig. 1 a Cystic fibrosis (CF) destroys the lungs, and the patients die of pulmonary disease, generally in childhood. b The autosomal-recessive mutations alter the membrane-distribution and function of a chloride channel, cystic fibrosis transmembrane regulator (CFTR). CFTR is a member of the ATP-binding cassette (ABC) transporter superfamily that contains two membrane spanning domains (MSDs), two cytosolic nucleotide binding domains (NBDs), and a regulatory (R) domain.

\section{B CFTR}

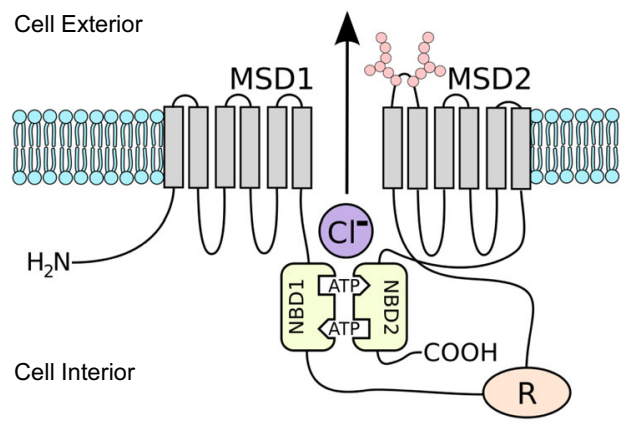

the treatment of cancer, rheumatoid arthritis, acne, psoriasis, and CF [7]. In an earlier study, Mcilroy et al. showed that fenretinide-mediated retinoic acid receptor signaling and inhibition of ceramide biosynthesis regulates adipogenesis, lipid accumulation, mitochondrial function, and nutrient stress signaling in adipocytes and adipose tissue. Their findings suggested that fenretinide utilizes retinoid-acid-receptor (RAR)dependent and independent pathways to regulate cell biology that could be relevant to the current results [8].

The authors of this month's J Mol Med Garić et al. show a restoration of CFTR expression when aberrant levels of specific ceramides, as determined by means of a mass spectrometry assay, are reduced to normal [9]. Furthermore, they demonstrate a correlation between CFTR protein expression and regulation of specific ceramide levels. They first demonstrated that very-long-chain ceramide species are reduced, while long-chain ceramide expression (14-18 carbons) is increased in Cftr knockout mice, compared to controls [9]. Petrache et al. had suggested earlier that increased long-chain ceramides lead to increased inflammation in the lungs [10]. Garic et al. then treated Cftr knockout mice with fenretinide for 4 weeks [9]. The treatment brought about downregulation of long-chain ceramides, while actually increasing very-longchain ceramides. The authors also treated $\mathrm{CF}$ patients with fenretinide. The treatment had heterogeneous effects so that
(Image taken from https://de.wikipedia.org) c CFTR failure alters all exocrine glands; thus, their mucous product is sticky and results in much more infections because of altered biofilms. The local production of certain ceramides through degradation of sphingomyelins has been implicated in promoting infection with Pseudomonas aeruginosa. (PC phosphocholine, ASM acid syphingomyelinase, AC acid ceramidase, FA fatty acids)

the 15 patients were divided into responders or nonresponders. Nevertheless, $300 \mathrm{mg}$ daily of fenretinide in these patients appeared to normalize the balance between long-chain and very-long-chain ceramides. Non-CF persons showed no effects of these treatments, nor did placebo treatment exert any effect.

The authors also found that pulmonary epithelial cells homozygous for CFTR $\triangle \mathrm{F} 508$ also exhibited a favorable ceramide expression response after exposure to fenretinide [9]. In these experiments, authors used a lentiviral vector encoding functional CFTR protein. The investigators again found reduced long-chain ceramide expression and increased verylong-chain ceramide expression. Interestingly, treatment with zinc also influenced the results in a similar fashion. The authors suggest that transcriptional regulation could be responsible for their results.

Seitz et al. [11] indicate that ceramide accumulates in the airway epithelium of CF and ceramide synthase 2 (CerS2)deficient mice, which respond to the lack of very-long-chain (C22-C24-) ceramides with a profound compensatory increase of long-chain (mainly C16-) ceramides. In contrast, sphingosine is present in healthy airways and is almost completely absent from diseased or deficient epithelial cells. Both sphingolipids are crucially involved in the high susceptibility to infection of $\mathrm{CF}$ and CerS2-deficient mice, as 
indicated by findings showing that the normalization of ceramide and sphingosine levels rescue these mice from acute infection with $P$. aeruginosa. Both sphingolipids could be crucially involved in the high susceptibility to infection in $\mathrm{CF}$ and CerS2-deficient mice, as indicated by findings showing that the normalization of ceramide and sphingosine levels rescue these mice from acute infection with P. aeruginosa.

How could fenretinide work? Fenretinide is a peroxisome proliferator-activated receptor- $\gamma$ (PPAR $\gamma$ ) ligand. Interestingly, PPAR $\gamma$ has been implicated as a therapeutic target in CF and its function has been known to be severely reduced in CF [12]. The PPAR $\gamma$ agonist, rosiglitazone was withdrawn from the market. However, pioglitazone remains available in some countries. Interestingly, PPAR $\gamma$ agonists were recently shown to reduce biofilm formation by $P$. aeruginosa [13]. Intriguing information for CF patients, whose outlook appears to be improving.

Respectfully,

Friedrich C. Luft

\section{References}

1. O'Sullivan BP, Freedman SD (2009) Cystic fibrosis. Lancet 373: 1891-1904

2. Bobadilla JL, Macek M Jr, Fine JP, Farrell PM (2002) Cystic fibrosis: a worldwide analysis of CFTR mutations - correlation with incidence data and application to screening. Hum Mutat 19:575606

3. Saiman L (2004) Microbiology of early CF lung disease. Paediatr Respir Rev 5 Suppl A: S367-369

4. Hannun YA, Obeid LM (2008) Principles of bioactive lipid signalling: lessons from sphingolipids. Nat Rev Mol Cell Biol 9:139-150
5. Tibboel J, Reiss I, de Jongste JC, Post M (2014) Sphingolipids in lung growth and repair. Chest 145:120-128

6. Grassme H, Riethmuller J, Gulbins E (2013) Ceramide in cystic fibrosis. Handb Exp Pharmacol doi: 10.1007/978-3-7091-1511-4 13: $265-274$

7. Guilbault C, De Sanctis JB, Wojewodka G, Saeed Z, Lachance C, Skinner TA, Vilela RM, Kubow S, Lands LC, Hajduch M et al (2008) Fenretinide corrects newly found ceramide deficiency in cystic fibrosis. Am J Respir Cell Mol Biol 38:47-56

8. Mcllroy GD, Tammireddy SR, Maskrey BH, Grant L, Doherty MK, Watson DG, Delibegovic M, Whitfield PD, Mody N (2016) Fenretinide mediated retinoic acid receptor signalling and inhibition of ceramide biosynthesis regulates adipogenesis, lipid accumulation, mitochondrial function and nutrient stress signalling in adipocytes and adipose tissue. Biochem Pharmacol 100:86-97

9. Garić D, De Sanctis JB, Wojewodka G, Houle D, Cupri S, AbuArish A, Hanrahan JW, Hajduch M, Matouk E, Radzioch D (2017) Fenretinide differentially modulates the levels of long- and very long-chain ceramides by downregulating Cers5 enzyme: evidence from bench to bedside. J Mol Med (Berl) DOI 10.1007/s00109017-1564-y

10. Petrache I, Kamocki K, Poirier C, Pewzner-Jung Y, Laviad EL, Schweitzer KS, Van Demark M, Justice MJ, Hubbard WC, Futerman AH (2013) Ceramide synthases expression and role of ceramide synthase-2 in the lung: insight from human lung cells and mouse models. PLoS One 8:e62968

11. Seitz AP, Grassme H, Edwards MJ, Pewzner-Jung Y, Gulbins E (2015) Ceramide and sphingosine in pulmonary infections. Biol Chem 396:611-620

12. Dekkers JF, van der Ent CK, Kalkhoven E, Beekman JM (2012) PPARgamma as a therapeutic target in cystic fibrosis. Trends Mol Med 18:283-291

13. Bedi B, Maurice NM, Ciavatta VT, Lynn KS, Yuan Z, Molina SA, Joo M, Tyor WR, Goldberg JB, Koval M et al (2017) Peroxisome proliferator-activated receptor-gamma agonists attenuate biofilm formation by Pseudomonas aeruginosa. FASEB J DOI 10.1096/fj. 201700075R 